\title{
Ultra-Wideband Array in PCB for Millimeter-Wave 5G and ISM
}

\author{
Markus H. Novak, John L. Volakis \\ ElectroScience Laboratory, The Ohio State University \\ Columbus, OH, USA
}

\author{
Félix A. Miranda \\ NASA Glenn Research Center \\ Cleveland, $\mathrm{OH}$, USA
}

\begin{abstract}
Next generation 5G mobile architectures will take advantage of the millimeter-wave spectrum to deliver unprecedented bandwidth. Concurrently, there is a need to consolidate numerous disparate allocations into a single, multi-functional array. Existing arrays are either narrow-band, prohibitively expensive or cannot be scaled to these frequencies. In this paper, we present the first ultra-wideband millimeter-wave array to operate across the six 5G and ISM bands spanning 24-71 GHz. Critically, the array is realized using low-cost PCB. The design concept and optimized layout are presented, and fabrication and measurement considerations are discussed.
\end{abstract}

\section{INTRODUCTION}

Communication platforms (whether cell phones, autonomous vehicles, satellites, etc.) are increasingly multifunctional, and require access to widely separated portions of the spectrum. Indeed, a cellphone today may operate in as many as 7 different bands. This leads to redundancy and inefficiency, as independent systems are required for each frequency band. Concurrently, the ever-growing need for high data rates has led to the licensing of the millimeter-wave spectrum.

In spite of the growing needs for more bandwidth, the fragmentation of the electromagnetic spectrum continues. As Table I shows, the existing unlicensed Industrial, Scientific, Medical (ISM) bands and the recently allocated Fifth Generation Mobile (5G) bands, are widely distributed in and around the millimeter-wave spectrum [1]. Together, these allocations span nearly $50 \mathrm{GHz}$.

TABLE I

Allocated Millimeter-WaVe 5G AND ISM Bands

\begin{tabular}{|c|c||c|c|}
\hline Allocation & Frequency $(\mathrm{GHz})$ & Allocation & Frequency $(\mathrm{GHz})$ \\
\hline ISM & $24-25$ & $5 \mathrm{G}$ & $38.6-40$ \\
\hline $5 \mathrm{G}$ & $27.5-28.35$ & ISM & $57-64$ \\
\hline $5 \mathrm{G}$ & $37-38.6$ & $5 \mathrm{G}$ & $64-71$ \\
\hline
\end{tabular}

Importantly, free-space path loss in the millimeter-wave bands is significant. Therefore $5 \mathrm{G}$ architectures require high gain phased arrays to overcome losses in the link budget. Because of cost and space limitations, it is not feasible to employ several such arrays to cover the 5G and ISM bands in Table I. Instead, it is necessary to consolidate all six noted $5 \mathrm{G}$ and ISM

This work was supported by a NASA Space Technology Research Fellowship, under grant \#NNX13AL48H. millimeter-wave bands into a single multi-functional aperture. Simultaneously, true mass-market compatibility implies lowcost PCB implementation, and high efficiency.

Although many millimeter-wave arrays are found in the literature, they are invariably either narrow-band [2] and/or require expensive wafer-scale fabrication [3]. Moreover, onchip antennas suffer from low efficiency [4]. Conversely, thin ultra-wideband (UWB) arrays such as coupled or connected arrays [5] cannot be scaled to higher frequencies. The highest frequency UWB arrays operate at 7-21 GHz [6], 8$40 \mathrm{GHz}$ [3], and 9-49 GHz (simulation only) [7].

In this paper, we present a planar, UWB array (see Fig. 1) that provides continuous coverage from 24-72 GHz. Being compatible with standard PCB manufacturing, the array is extremely low-cost and can take advantage of low-loss laminates. In the following, we discuss the design approach and performance. Fabrication and measurement considerations are also provided.

\section{DESIGN CONCEPT}

The proposed array is based on the tightly coupled dipole array (TCDA) [5]. Specifically, it employs capacitive coupling between neighboring elements to cancel the inductance from the nearby groundplane. The TCDA element is further integrated with a balun within the volume of the array [8] [10].The additional reactance from this electrically small balun further serves as a higher order impedance matching network, and constitutes a critical part of the design process. However, designs such as those in [7], [10], [11] are associated with complex, through-plane feeds. Therefore, they cannot be scaled up to millimeter-wave. Conversely, planarized feeds such as [8] suffer from reduced bandwidth.

While the TCDA array element are readily scaled to higher frequencies, the complexity of the feeding network represents the primary challenge for adapting these to millimeter waves. In a planar PCB implementation, metalized vias represent the primary through-plane element from which the balun is constructed. These vias have diameters of at least $150 \mu \mathrm{m}$, at a pitch $>400 \mu \mathrm{m}$ - a significant fraction of the wavelength at $71 \mathrm{GHz}$

We propose here a TCDA array with a highly compact element and feeding network, comprising only three vias and two metalized layers, as shown in the physical-equivalent circuit diagram in Fig. 2. To satisfy the condition $Z_{\text {short }}>>$ 


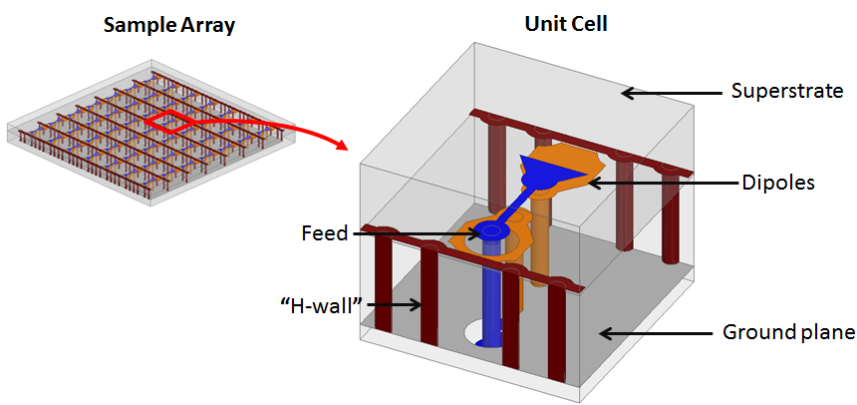

Fig. 1. Illustration of a finite array and the proposed unit cell design.
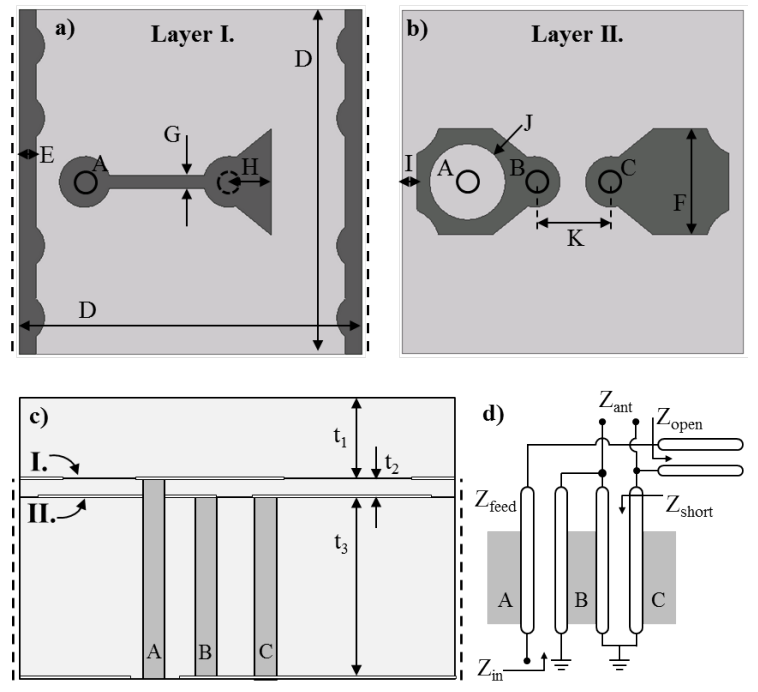

Fig. 2. Design layout and parameters of the new array element: a) top layer, b) bottom layer, c) side view, d) equivalent circuit. Placement of the conducting $\mathrm{H}$-wall is indicated with dashed lines in a) and c).

$Z_{\text {open }}$ [12], vias are used to form high-impedance twin-wire transmission lines for the feed and short-circuit stub. The open stub is also shown, and has a low impedance, $Z_{\text {open }}$. While the shielded stripline traces used in [7], [10] help to prevent spurious modes and cross-polarized radiation, this compromise allows the feed to be realized at millimeter-wave frequencies.

\section{IMPLEMENTATION}

The proposed array unit cell layout and design parameters are shown in Fig. 2. As noted, the exposed nature of the feeding network introduces the risk of spurious modes inside the substrate. When situated in an array environment, one such common-mode resonance can indeed be observed, as depicted in Fig. 3. Fortunately, only slight modifications are required to mitigate the onset of this resonance, caused an imbalance in the magnitude of the differential currents flowing on the dipole pins, B and C (Fig. 2). To guide us in avoiding feed resonances, we note that the field distribution resembles a waveguide $\mathrm{TE}_{10}$ mode between grounded conductors. In fact, for two conductors separated by distance $D$, we can estimate

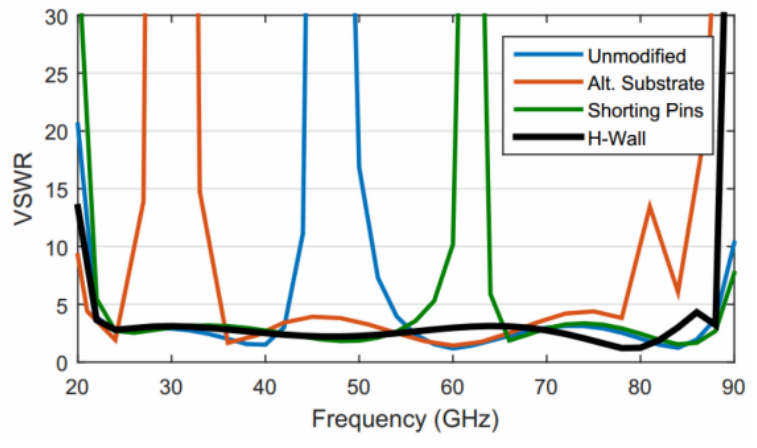

Fig. 3. Array response demonstrating common mode resonance for the unmodified feed design (blue), high $\epsilon_{r}$ substrate (red), shorting pin (green), and with the conducting wall parallel to the element H-plane (black).

TABLE II

OPTIMIZED DESIGN PARAMETER VALUES

\begin{tabular}{|c|c||c|c||c|c|}
\hline Parameter & $(\mathrm{mil})$ & Parameter & $(\mathrm{mil})$ & Parameter & $($ mil $)$ \\
\hline$\varnothing \mathrm{A}$ & 6 & $\varnothing \mathrm{B}$ & 6 & $\varnothing \mathrm{C}$ & 6 \\
\hline $\mathrm{D}$ & 81 & $\mathrm{E}$ & 4 & $\mathrm{~F}$ & 25 \\
\hline $\mathrm{G}$ & 3 & $\mathrm{H}$ & 16 & $\mathrm{I}$ & 3.5 \\
\hline$\varnothing \mathbf{J}$ & 18 & $\mathrm{~K}$ & 12 & $t_{1}$ & 20 \\
\hline$t_{2}$ & 4 & $t_{3}$ & 36 & & \\
\hline
\end{tabular}

the resonant frequency using the well-known relation:

$$
f_{r}=\frac{c}{2 D \sqrt{\epsilon_{r}}}
$$

For $D=2.2 \mathrm{~mm}$, this gives $f_{r}=46 \mathrm{GHz}$, corresponding to the central peak observed in Fig. 3. Likewise, we see in Fig. 3 that the application of shorting pins between neighboring elements or use of alternate substrate materials can alter the $D \sqrt{\epsilon_{r}}$ term. Both of these approaches can be used to manipulate the resonant frequency.

The occurrence of this resonance can be pushed higher, and ultimately out of the band, by replacing the single shorting pin with a continuous conducting wall, perpendicular to the primary dipole current (black curve, Fig. 3). In practice, this can be accomplished using a fence of vias or by implementing dual polarizations, similar to [6]. It is noted that if the fence of vias is used, the vias must be electrically connected above the groundplane to prevent monopole radiation.

With the above factors in mind, the optimized design was developed, and the parameter values of Fig. 2 are given in Table II. This optimal element was used in an infinite array to evaluate its VSWR performance. The VSWR is shown in Fig. 4 for broadside and scanning cases, with the frequency bands of Table I highlighted. We note that the design is well matched across the desired $24-71 \mathrm{GHz}$ band. Specifically, broadside VSWR $<2.2$. Also, for scans to $\pm 45^{\circ}$ VSWR $<2$ in the E-plane and VSWR $<3$ in the H-plane. Additionally, the highly symmetric nature of the element leads to very low cross-polarized gain $(<-50 \mathrm{~dB}$ in the principal planes). 


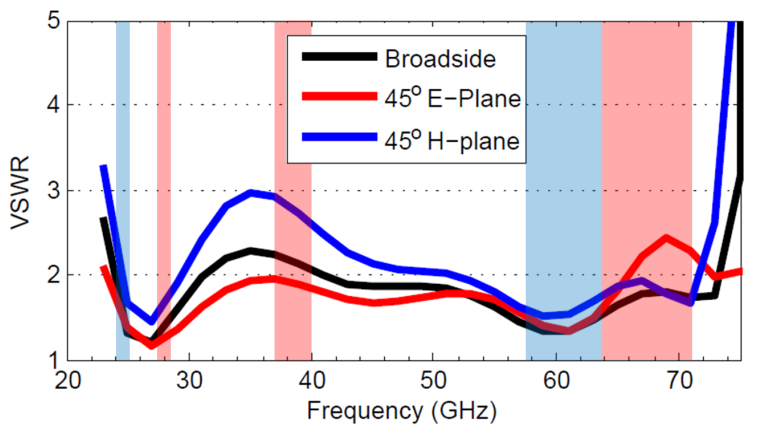

Fig. 4. VSWR performance of the infinite array using the optimized array element design, with the values given in Table II. The 5G and ISM allocations given in Table I are highlighted for reference.

\section{FABRICATION \& MEASUREMENT}

Several prototype arrays are currently being fabricated through commercial PCB vendors, using the dimensions given in Table II. However, at these frequencies it is also challenging to conduct pattern or return loss measurements without including a beamforming network. Specifically, to measure the performance of individual elements within the array we must use a test fixture to interface with the VNA. However, the large size of the test fixture (such as that illustrated in Fig. 5) distorts the response of the embedded test article and must therefore be compensated for. A de-embedding network is shown in Fig. 5. We observe that the measured reflection coefficient, $\Gamma_{\text {Meas }}$ is related to the antenna reflection coefficient, $\Gamma_{D U T}$, according to

$$
\Gamma_{\text {Meas }}=S_{11}+\frac{S_{12} S_{21} \Gamma_{D U T}}{1-S_{22} \Gamma_{D U T}} .
$$

From this, we can then extract $\Gamma_{D U T}$, given by

$$
\Gamma_{D U T}=\frac{S_{11}-\Gamma_{\text {Meas }}}{S_{11} S_{22}-S_{12} S_{21}-S_{22} \Gamma_{\text {Meas }}} .
$$

That is, by isolating the two-port scattering parameters $S_{i j}$ of the test fixture, the desired measurement can be deembedded from the raw data. These scattering parameters can be determined by measuring identical test fixtures, with the DUT is replaced with open, short, and matched loads. For these cases, $\Gamma_{D U T}=1, \Gamma_{D U T}=-1$, and $\Gamma_{D U T}=0$, respectively. As $S_{12}=S_{21}$, the open, short, and matched test allow determination of $S_{i j}$. Results of these measurements will be discussed at the conference.

\section{CONCLUSION}

For the first time, we presented a low cost, UWB, millimeter-wave array that employs a simplified feeding network requiring only three vias and two metal layers. The design process and a new array design was presented demonstrating low VSWR across six 5G and ISM bands, spanning 24$71 \mathrm{GHz}$. Unlike designs found in the literature, the presented work accounts for feature size and via spacing limitations, making its fabrication possible using large-scale and low-cost PCB approaches. Fabrication results and measurements will be presented at the conference.
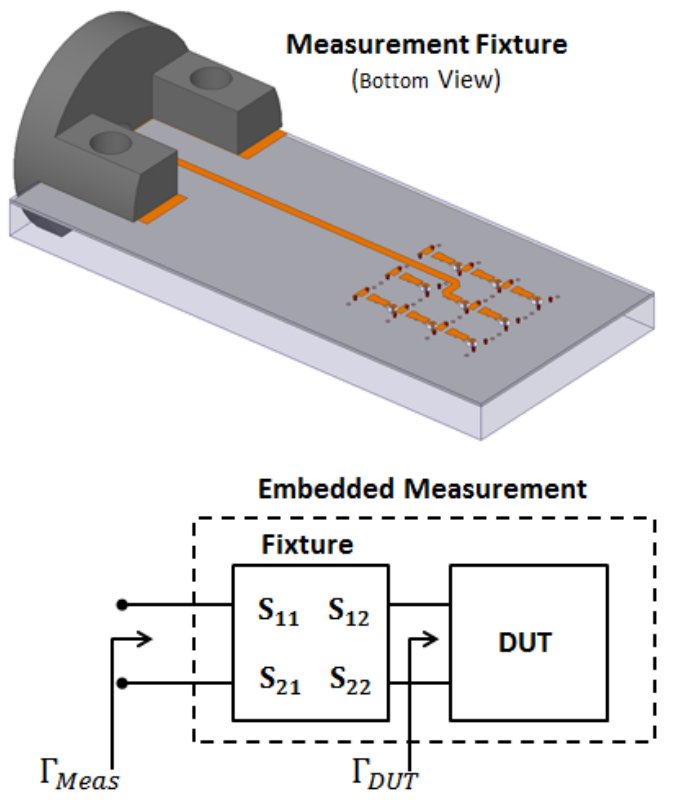

Fig. 5. Rendering of the test fixture, including $1.85 \mathrm{~mm}$ coaxial connector and microstrip input trace feeding a single element of the $3 \times 3$ array (top), and S-parameter model of the embedded measurement setup (bottom).

\section{REFERENCES}

[1] “FCC-16-89," Federal Communications Commission, July 2016.

[2] G. Rankin, A. Tirkel, and A. Leukhin, "Millimeter wave array for UAV imaging MIMO radar," in Intl. Radar Symp., June 2015, pp. 499-504.

[3] T. Durham, K. Vanhille, C. Trent, K. Lambert, and F. Miranda, "Design of an 8-40 GHz antenna for WISM," in IEEE Ant. and Prop. Intl. Symp., July 2015, pp. 1999-2000.

[4] S. Alalusi and R. Brodersen, "A $60 \mathrm{GHz}$ phased array in CMOS," in Custom Integrated Circuits Conference, 2006. CICC'06. IEEE. IEEE, 2006, pp. 393-396.

[5] B. A. Munk, Finite antenna arrays and FSS. Wiley, 2003.

[6] S. S. Holland and M. N. Vouvakis, "The planar ultrawideband modular antenna (PUMA) array," IEEE Trans. Ant. and Propag., vol. 60, no. 1 pp. 130-140, 2012.

[7] M. H. Novak, F. A. Miranda, and J. L. Volakis, "Ultra-wideband phased array antennas for satellite communications up to $\mathrm{Ku}$ - and $\mathrm{Ka}$-band," IEEE Trans. Ant. and Propag., In review.

[8] J. A. Kasemodel, C. C. Chen, and J. L. Volakis, "Wideband planar array with integrated feed and matching network for wide-angle scanning," IEEE Transactions on Antennas and Propagation, vol. 61, no. 9, pp. 4528-4537, Sept 2013.

[9] W. F. Moulder, K. Sertel, and J. L. Volakis, "Superstrate-enhanced ultrawideband tightly coupled array with resistive FSS," IEEE Trans. Ant. and Propag., vol. 60, no. 9, pp. 4166-4172, 2012.

[10] J. Doane, K. Sertel, and J. L. Volakis, "A wideband, wide scanning tightly coupled dipole array with integrated balun (TCDA-IB)," IEEE Trans. Ant. and Propag., vol. 61, no. 9, pp. 4538-4548, 2013.

[11] D. Cavallo, A. Neto, G. Gerini, and A. Micco, "A novel printed-circuitboard feeding structure for common-mode rejection in wide-scanning connected arrays of dipoles," in EuCAP 2010, April 2010, pp. 1-4.

[12] D. Bartholomew, "Optimum design for a broadband microstrip balun," Electronics Letters, vol. 13, no. 17, pp. 510-511, August 1977. 